Lutz Nasdala · Marita Wenzel · Gerhard Vavra

Gert Irmer · Thomas Wenzel · Bernd Kober

\title{
Metamictisation of natural zircon: accumulation versus thermal annealing of radioactivity-induced damage
}

Published online: 19 July 2002

(c) Springer-Verlag 2002

\section{Contrib Mineral Petrol (2001) 141:125-144}

Due to unfortunate mistakes when looking up $\alpha$-energies from the literature, parts of the calculations in Table 2 were done with incorrect values. The simulations were redone. The corrected table is given below:
The online version of the original article can be found at http:// dx.doi.org/10.1007/s004100000235

L. Nasdala $(\bowtie) \cdot T$. Wenzel

Institut für Geowissenschaften - Mineralogie,

Johannes Gutenberg-Universität,

55099 Mainz, Germany

E-mail: nasdala@mail.uni-mainz.de

Tel: + 49-6131-3924781

Fax: + 49-6131-3923070

M. Wenzel

Institut für Festkörperphysik,

Technische Universität, 64289 Darmstadt, Germany

G. Vavra

Institut für Mineralogie, Eberhard Karls-Universität,

Wilhelmstraße 56, 72074 Tübingen, Germany

G. Irmer

Institut für Theoretische Physik,

TU Bergakademie Freiberg,

Bernhard-von-Cotta-Straße 4,

09596 Freiberg/Sa., Germany

B. Kober

Institut für Mineralogie, Ruprecht-Karls-Universität,

Im Neuenheimer Feld 236, 69120 Heidelberg, Germany

Present address: M. Wenzel

Schott, Postfach 2480,

55014 Mainz, Germany

\section{Reference}

Firestone RB, Shirley VS (1996) Table of isotopes 2. Wiley, New York 
Table 2. Results of Monte Carlo simulations: Average ranges of ${ }^{4} \mathrm{He}$ cores and recoiled heavy daughter nuclei and average numbers of atomic vacancies created per $\alpha$-decay event

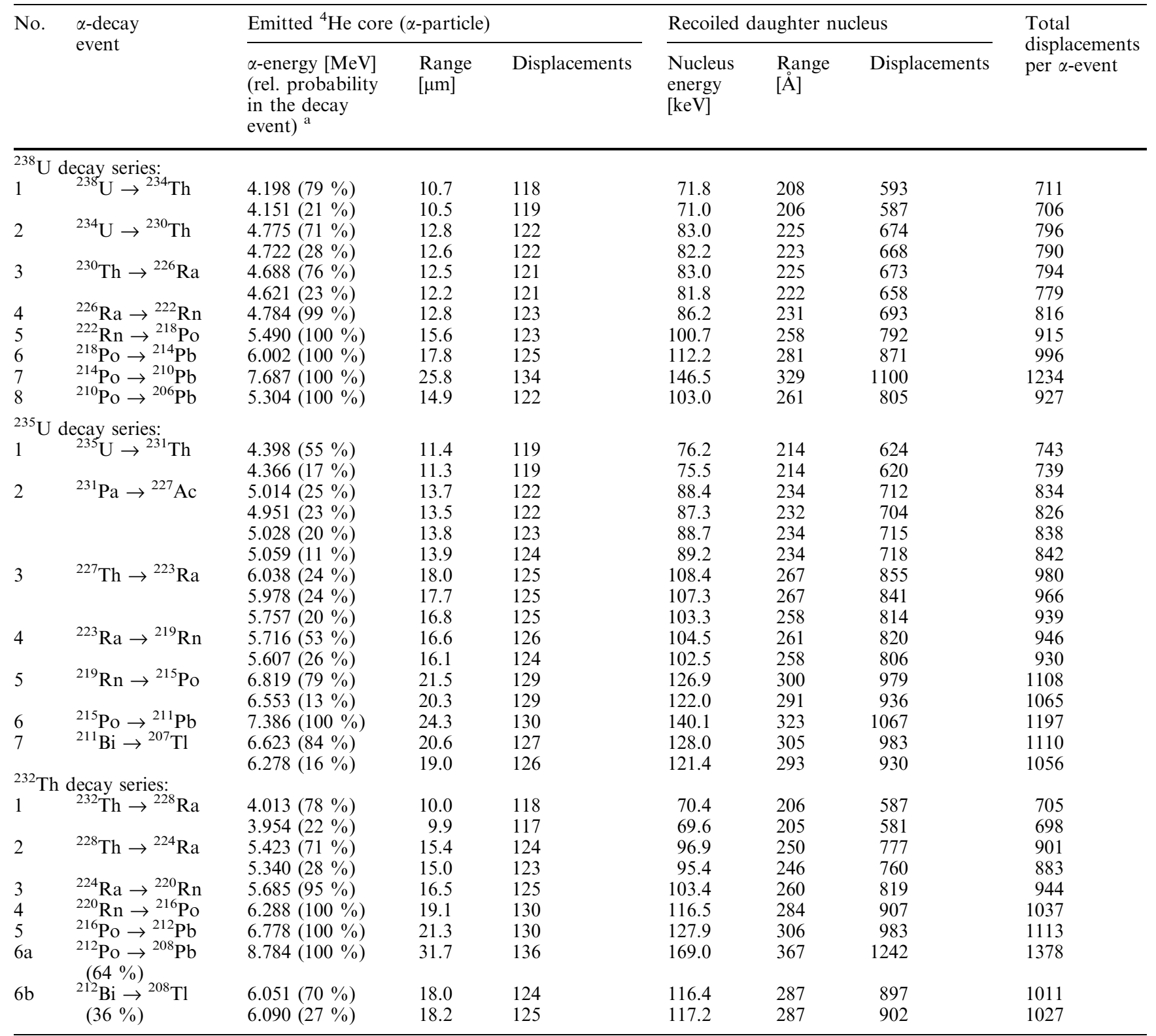

${ }^{a}$ Data from Firestone and Shirley (1996), rounded values. Simulations were only done for $\alpha$-energies with relative probabilities of $>10 \%$ in the branching paths 